\title{
Optic disc drusen in children: morphologic features using EDI-OCT
}

\author{
Filipa Jorge Teixeira $\mathbb{D}^{1,2} \cdot$ Raquel Esteves Marques $\mathbb{C}^{1,2} \cdot$ Sofia Sousa Mano ${ }^{1,2} \cdot$ Rita Couceiro $^{3} \cdot$ Filomena Pinto $^{1,2}$
}

Received: 17 February 2019 / Revised: 20 September 2019 / Accepted: 30 September 2019 / Published online: 19 November 2019

(c) The Author(s), under exclusive licence to The Royal College of Ophthalmologists 2019

\begin{abstract}
Aims This study aimed to investigate morphologic features of optic disc drusen (ODD) and peripapillary hyperreflective ovoid mass-like structures (PHOMS) in children, using enhanced depth imaging optical coherence tomography (EDI-OCT). It also assessed if the presence of these features were associated with decreased peripapillary retinal nerve fibre layer (RNFL) thickness.

Methods Retrospective observational study of children with ODD. All subjects underwent complete ophthalmic examination and multimodal imaging. ODD were identified on EDI-OCT as circumscribed hyporeflective spheroidal elements located in front of lamina cribrosa, fully or partially surrounded by a hyperreflective border. PHOMS were identified as hyperreflective ovoid structures located in the peripapillary circumference. Both associations between ODD and RNFL loss and PHOMS and RNFL loss were tested using chi-squared test.

Results In total, 38 eyes of 20 children were analysed. PHOMS were present in 90\% of patients. ODD and PHOMS were predominantly found in the nasal, superonasal and inferonasal sectors. A significant positive association was found between ODD and decreased RNFL thickness in the nasal $(p=0.02)$, superonasal $(p=0.05)$ and inferotemporal $(p=0.04)$ sectors. There was no significant association found with the presence of PHOMS.

Conclusion EDI-OCT allowed morphological analysis of ODD and PHOMS in children. Drusen were found to be distinct from PHOMS both in their appearance and impact on the RNFL. ODD are hyporeflective and appear on the ONH above the lamina cribrosa and were associated with decreased thickness of the RNFL. On the contrary, PHOMS are hyperreflective structures located around the ONH and were not associated with RNFL loss.
\end{abstract}

\section{Introduction}

Optic disc drusen (ODD) are acellular, calcified deposits located in optic nerve head $(\mathrm{ONH})$ [1]. In a recently published Danish population cohort study, that included 1304 children, the prevalence of ODD in children was found to be $1 \%$ [2].

ODD in children are typically bilateral and are more likely to be buried than in adults [3, 4]. When located near the surface, ODD can be directly visualised by fundus examination, presenting as an irregular lumpy appearance of the ONH [3]. Buried ODD are located closer to the

Filipa Jorge Teixeira

filipajorge.t@gmail.com

1 Hospital de Santa Maria, Lisboa, Portugal

2 Faculdade de Medicina, Clínica Universitária de Oftalmologia, Universidade de Lisboa, Lisboa, Portugal

3 Hospital de Vila Franca de Xira, Vila Franca de Xira, Portugal lamina cribrosa and can be difficult to detect. In fundus examination of buried ODD, the optic disc appears elevated, without cupping with blurred margins, although without obscuration of peripapillary retinal vessels $[3,5,6]$. It is hypothesised that ODD become visible with age, either because of drusen growth, increase in number, migration or due to an age-related thinning of the overlying retinal nerve fibre layer (RNFL) [3, 5].

Several ancillary tests, including ophthalmic ultrasound (US), orbital computed tomography (CT), fundus autofluorescence (FAF), fluorescein angiography (FA), and optical coherence tomography (OCT), have been used to identify ODD [7]. The role of OCT to diagnose ODD has expanded in the recent years, especially with the development of enhanced depth imaging OCT (EDI-OCT), which has its highest sensitivity near the inner sclera and is thereby excellent in visualising buried ODD [8-10]. In the last few years, there have been conflicting descriptions of ODD morphology on OCT. Generally the ODD were described as a signal-poor core mass surrounded by a hyperreflective margin [8-12]. However, other studies described ODD as 
hyperreflective structures or with both hypo- and hyperreflective internal reflectivity $[12,13]$. A critical appraisal regarding this description has been raised by some authors $[8,14]$. Hyperreflective mass-like structures, almost always located in the peripapillary circumference appear in patients with ODD although, they are also present in patients with intracranial hypertension $[8,15]$ or with optic nerve tumours [16]. In a recent consensus report from the Optic Disc Drusen Studies (ODDS) Consortium, these peripapillary hyperreflective ovoid mass-like structures, were termed PHOMS, and it was stated that until or unless there is histopathological evidence to the contrary, PHOMS should be excluded as a criteria for the OCT diagnosis of ODD [17].

The primary aim of this study was to investigate the morphologic features of ODD and PHOMS in children, using EDI-OCT, and assess if their presence is associated with a decrease in peripapillary RNFL thickness. Secondary outcomes included assessment of the differences in clinical and imaging findings comparing patients with visible and buried ODD.

\section{Materials and methods}

The research is a retrospective single-centre observational study. Patients under 18 years old and with the diagnosis of ODD were included. This study was conducted at the Department of Ophthalmology from Hospital Santa Maria in Lisbon, Portugal, with the approval of the Internal Review Board and was performed in accordance with the tenets of the Declaration of Helsinki. Informed consent was obtained from all patients.

All children had a full ophthalmologic examination including best-corrected visual acuity (BCVA), slit-lamp biomicroscopy and dilated fundus examination. Patients underwent imaging with optic disc stereo-photography, US, FAF and OCT of the macula and ONH. All OCT and US exams were obtained by the same experienced operator (F.P). Standard automated perimetry (Humphrey VF Analyzer, 24-2 SITA-FAST; Carl Zeiss Meditec, Inc, Dublin, CA) was performed in children who were able to cooperate.

Based on dilated fundus examination and fundus photography, eyes were classified with visible ODD if there were discrete or coalescent deposits within or at the margin of the optic disc, and imaging exams confirmed the presence of ODD. Eyes were classified as buried ODD if the optic disc did not have any clinically visible deposits and the margins of the disc were either normal, elevated and/or irregular, and imaging exams (FAF, OCT and/or US) confirmed the presence ODD.

\section{Structural analysis of ODD and ONH with OCT}

Imaging scans for FAF, SD and EDI-OCT were captured with the Heidelberg Spectralis OCT, software version 3.1 (Heidelberg Engineering, Heidelberg, Germany). The pupil was dilated with $10 \%$ phenylephrine and $1 \%$ tropicamide before examination. Patient's fixation was monitored by asking the patient to look at an internal light fixation target.

For peripapillary RNFL analysis, scans were acquired using a standardised $3.4 \mathrm{~mm}$ diameter circle around the optic disc. An automated algorithm identified the anterior and posterior aspects of the nerve fibre layer and calculated the average thickness and the thickness in six sectors: nasal, temporal, temporal superior, nasal superior, temporal inferior and nasal inferior. The Spectralis OCT software, Heidelberg Explorer (version 5.3; Heidelberg Engineering Co., Heidelberg, Germany), was used for automatic segmentation and to calculate RNFL thickness. Manual adjustment of upper and lower boundary lines when position was misplaced was performed by the examiner. The thickness in each sector was segregated into three categories: the lower one percentile, which had the descriptor 'outside normal limits'; a range from the second through fifth percentile, termed 'borderline'; and the remaining upper $95 \%$ that was considered 'within normal limits'. Patients with borderline and outside normal limits were considered to have RNFL thickness loss.

FAF was performed with confocal scanning laser ophthalmoscope (Spectralis HRA, Heidelberg Engineering, Heidelberg, Germany) with 100 consecutive frames centred at the $\mathrm{ONH}$.

Radial scans of the ONH were performed with EDIOCT. The radial scanning pattern centred on the ONH was used to obtain eight angularly equidistant radial B-scans $(30 \times 30$ degrees a part), each $6 \mathrm{~mm}$ long. Each radial Bscan was averaged from 100 frames with 1024 A-scans per B-scan. The internal image registration and tracking software was used to significantly reduce the effects of eye movements [18]. EDI-OCT images were carefully reviewed for scleral canal size measurements and to detect the presence of ODD by the same experienced observer (F.P) who was masked for the patients' clinical information. The scleral canal size was manually measured as the mean of the largest vertical and horizontal opening of Bruch's membrane. Detection and structural analysis of ODD was done according to ODDS Consortium recommendations [17], including: (1) ODD are always located above the lamina cribrosa; (2) ODD always have a hyporeflective core; (3) ODD are often but not always seen with hyperreflective margins, most prominent superiorly; (4) hyperreflective horizontal lines might represent early ODD but should not be diagnosed as ODD; and (5) PHOMS should not be 

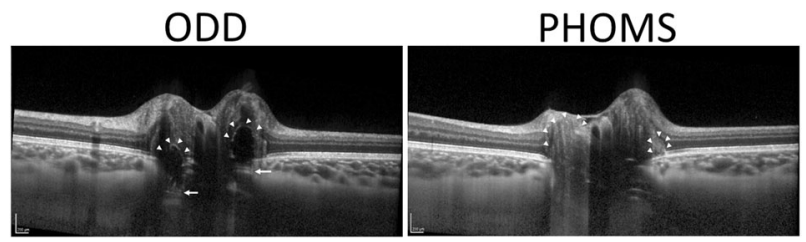

Fig. 1 Configuration of optic disc drusen (left) and PHOMS (right) on EDI-OCT. On the left ODD appear as large ovoid regions of lower reflectivity (arrowheads), with hyperreflective margins. Please note hyperreflective horizontal lines in deep location of the scleral canal (arrows). On the right PHOMS present as hyperreflective ovoid masses (arrowheads) around ONH. Abbreviations: EDI-OCT enhanced depth imaging optical coherence tomography, ODD optic disc drusen, PHOMS peripapillary hyperreflective ovoid mass-like structures

diagnosed as ODD. Please see Fig. 1 to illustrate the detection of ODD and PHOMS on EDI-OCT.

\section{Statistical analysis}

Snellen BCVA measurements were converted to logarithm of the minimum angle of resolution visual acuity (logMAR) equivalents for data analysis.

Mean and standard deviations or median and interquartile ranges (skewed distributions) were reported for continuous variables. Univariate comparisons between treatment groups were performed with an independent sample student's $t$ test for continuous normally distributed variables and the chi-square test or Fisher's exact tests (expected count $<5$ ) were used for categorical variables. A $p$ value of 0.05 or less was considered statistically significant.

Statistical analysis was performed with SPSS (version 25.0, SPSS, Chicago, IL, USA).

\section{Results}

We retrospectively reviewed 38 eyes of 20 children with ODD. In $90 \%$ of the cases ODD were bilateral. Among the 20 eligible patients, one (5\%) was accidently diagnosed with ODD after performing a FAF as part of retinitis pigmentosa follow-up; and in 19 (95\%) patients ODD were diagnosed as part of the investigation of optic discs with blurred margins and/or obvious visualisation of ODD on fundus examination. Obvious visualisation of ODD was detected in 8 eyes (21\%) and the remaining 30 eyes (79\%) had buried ODD on ophthalmoscopy.

\section{Comparison of visible and buried ODD}

Differences in clinical and imaging findings were compared between patients with visible (visible by ophthalmoscopy/ fundus photography) and buried ODD (not visible by ophthalmoscopy/fundus photography) and are depicted in Table 1.
Table 1 Clinical and imaging features of patients with optic disc drusen: visible $v s$. buried

\begin{tabular}{|c|c|c|c|}
\hline & $\begin{array}{l}\text { Visible ODD } \\
(n=8)\end{array}$ & $\begin{array}{l}\text { Buried ODD } \\
(n=30)\end{array}$ & $p$ value \\
\hline Age, years $($ mean $\pm S D)$ & $14 \pm 4$ & $11 \pm 4$ & 0.11 \\
\hline Female:male ratio, female $\%$ & $4: 4(50 \%)$ & $23: 7(77 \%)$ & 0.14 \\
\hline $\begin{array}{l}\text { BCVA (LogMAR) (mean } \pm \\
\text { SD) }\end{array}$ & $0.00 \pm 0.00$ & $0.03 \pm 0.05$ & 0.11 \\
\hline $\begin{array}{l}\text { Spherical equivalent }(D) \\
(\text { mean } \pm \text { SD) }\end{array}$ & $-1.41 \pm 0.69$ & $-0.46 \pm 1.81$ & 0.16 \\
\hline $\begin{array}{l}\text { Mean scleral canal diameter } \\
\text { ratio V:H }(\mu \mathrm{m})\end{array}$ & $1788: 1675$ & $1561: 1436$ & $<0.01$ \\
\hline Visual field defects, $n(\%)$ & $2(25 \%)$ & $5(17 \%)$ & 0.04 \\
\hline $\begin{array}{l}\text { ODD related complications, } \\
n(\%)\end{array}$ & $1(13 \%)$ & $2(7 \%)$ & 0.60 \\
\hline ODD EDI-OCT, $n(\%)$ & $8(100 \%)$ & $30(100 \%)$ & - \\
\hline PHOMS EDI-OCT, $n(\%)$ & $6(75 \%)$ & $28(93 \%)$ & 0.13 \\
\hline $\begin{array}{l}\text { Fundus auto-fluorescence, } \\
n(\%)\end{array}$ & $4(50 \%)$ & $15(50 \%)$ & 0.65 \\
\hline $\begin{array}{l}\text { Ophthalmic ultrasound, } \\
n(\%)\end{array}$ & $8(100 \%)$ & $24(80 \%)$ & 0.17 \\
\hline $\begin{array}{l}\text { RNFL thickness, } \mu \mathrm{m} \\
(\mathrm{mean} \pm \mathrm{SD})\end{array}$ & $104 \pm 27$ & $107 \pm 26$ & 0.80 \\
\hline
\end{tabular}

$B C V A$ best-corrected visual acuity, $D$ dioptres, EDI-OCT enhanced depth imaging optical coherence tomography, $P H O M S$ peripapillary hyperreflective ovoid mass-lie structures, $R N F L$ retinal nerve fibre layer, $O D D$ optic disc drusen, $S D$ standard deviation, $V: H$ vertical: horizontal

Patients in the buried drusen group were younger (mean 11 years) than patients with visible ODD (mean 14 years), $p=0.11$. Female gender was more common in the buried ODD group (77\%), compared with the visible ODD group $(50 \%), p=0.14$. Patients with visible ODD were more myopic $(p=0.16)$. Patients with visible drusen disclosed statistically significant larger horizontal and vertical scleral canal diameters $(p<0.01)$.

Regarding imaging diagnosis, ophthalmic US detected all patients with visible ODD and $80 \%$ of patients with buried ODD, $p=0.17$. FAF showed a low diagnostic rate, identifying only $50 \%$ of the cases in both groups, $p=0.65$. PHOMS were more frequently found in patients with buried ODD (75\%) compared with patients with visible ODD (93\%), $\quad p=0.13$. Mean peripapillary RNFL thickness was slightly thinner in patients with visible ODD $(104 \mu \mathrm{m})$ compared with patients with buried ODD $(107 \mu \mathrm{m})$, $p=0.80$.

Twelve patients $(60 \%)$ were not able to cooperate in VF testing. Visual field defects were more common in patients with visible ODD (25\%) compared with patients with buried ODD $(17 \%), p=0.04$. Eight patients performed VF tests, and in nine eyes $(56 \%)$ VF were normal and in seven eyes $(44 \%)$ scotomas were present including: nasal step (four eyes), arcuate temporal inferior (two eyes) and 
temporal superior (one eye) and blind spot enlargement (one eye).

Complications related to ODD occurred in three patients $(15 \%)$, all cases were unilateral peripapillary haemorrhages with retinal and subretinal involvement. Two of these patients had buried ODD (6-year-old female and 17-yearold male) and a 17-year-old female had visible ODD. All patients presented with painless acute visual field loss. Subsequent scotomas (enlargement of blind spot in two cases, and nasal step in one patient) and decreased RNFL thickness were detected in this group of patients, although BCVA was not affected.

\section{Comparison of ODD and PHOMS}

The overall diagnostic rate of ODD was higher with EDIOCT (100\%), followed by ophthalmic US (84\%), FAF $(50 \%)$ and lastly by optic disc stereo-photograph (21\%). PHOMS were only detected with EDI-OCT and were found in $90 \%$ of the eyes (Table 2).

Using EDI-OCT a sector-based analysis was performed to detect where ODD and PHOMS were predominately located, results are depicted in Table 2. ODD were more commonly found in the nasal $(61 \%)$, inferonasal $(55 \%)$ and superonasal $(40 \%)$ sectors. PHOMS were also more common in the same sectors (superonasal $42 \%$, nasal $34 \%$ and inferonasal 34\%), for comparison please see Fig. 2.

Mean horizontal scleral canal diameter $(\mu \mathrm{m})$ was larger in children with ODD in the temporal sectors $(1632 \pm 222)$, and smaller in patients with ODD in the inferotemporal

Table 2 Detection of ODD and PHOMS with different imaging modalities. EDI-OCT detection of ODD and PHOMS: analysis by ONH sector

\begin{tabular}{lcll}
\hline Imaging exam & ODD $(n=38)$ & $\begin{array}{l}\text { PHOMS } \\
(n=34)\end{array}$ & $p$ value \\
\hline ONH stereo-photograph, & $8(21 \%)$ & - & \\
$n(\%)$ & $32(84 \%)$ & - & \\
Ophthalmic US, $n(\%)$ & $19(50 \%)$ & - & \\
Fundus auto-fluorescence, & 19 & \\
$n(\%)$ & $38(100 \%)$ & $34(89,5 \%)$ & \\
EDI-OCT, $n(\%)$ & & & \\
ONH sectors, $n(\%)$ & $23(61 \%)$ & $15(40 \%)$ & 0.96 \\
$\quad$ Nasal & $15(40 \%)$ & $16(42 \%)$ & 0.26 \\
$\quad$ Superonasal & $9(24 \%)$ & $12(32 \%)$ & 0.13 \\
Superotemporal & $9(24 \%)$ & $10(26 \%)$ & 0.58 \\
Temporal & $14(37 \%)$ & $12(32 \%)$ & 0.06 \\
Inferotemporal & $21(55 \%)$ & $13(34 \%)$ & 0.58 \\
$\quad$ Inferonasal & &
\end{tabular}

EDI-OCT enhanced depth imaging optical coherence tomography, PHOMS peripapillary hyperreflective ovoid mass-lie structures, $R N F L$ retinal nerve fibre layer, $O N H$ optic nerve head, $O D D$ optic disc drusen, $U S$ ultrasound
$(1507 \pm 144, p=0.20)$ and superotemporal $(1518 \pm 162$, $p=0.32$ ) sectors.

In this group of patients, the mean RNFL thickness was $106.6 \pm 25.8 \mu \mathrm{m}$ and in the nasal sector it was above reference values (Fig. 3). The RNFL thickness was outside/ borderline normal limits in 17 eyes (45\%). Only one sector with RNFL loss was found in eight eyes (21\%) and more than one sector in nine eyes $(24 \%)$. The sectors more predominantly affected were the inferotemporal (26\%), superotemporal $(24 \%)$ and nasal $(18 \%)$. The least involved sectors were the superonasal (13\%), inferonasal (13\%) and temporal $(5 \%)$. There was a significant positive association between the presence of ODD and the sectors where RNFL thickness was outside/borderline the normal limits [nasal $(p=0.02)$, superonasal $(p=0.05)$ and inferotemporal $(p=$ $0.03)]$. There was no significant association between the presence of PHOMS and RNFL loss (Table 3 and Fig. 2).

\section{Discussion}

This study analyses ODD features in paediatric population using EDI-OCT.

Drusen pathophysiology still remains unknown, although the most consensual theory describes their formation as a by-product of impaired axonal metabolism resulting in neuronal degeneration and accumulation of both intra- and extra-cellular calcium. Over time drusen increase in size and become more superficial [1, 3, 19] Consequently, in adults visible ODD are more frequent than in children [3, 4]. However no study has reported the prevalence of buried and visible ODD in paediatric population. As expected, we found a higher frequency of buried ODD (79\%) compared visible ODD (21\%). Furthermore, patients with visible ODD were older compared with those with buried ODD (mean $14 \pm 4$ vs $11 \pm 4$ years old, $p=0.11$ ). As previously documented in other studies [7], we found ODD to be more common in females.

In children with visible ODD spherical equivalent was slightly more myopic $(-1.41 \pm 0.69)$ compared with children with buried ODD $(-0.46 \pm 1.81), p=0.16$. It is possible that children with visible ODD due to older age present a higher myopic spherical equivalent, as myopic shift occurs during advancing age in childhood [20].

The current theory for drusen formation is explained by a genetic predisposition responsible for the dysplasia/narrowing of the scleral canal that creates nerve fibre compression [3, 5, 21]. OCT studies that compare scleral canal diameter of adult patients with ODD to healthy individuals report that patients with drusen have a wider canal $[13,21]$. On the contrary, children with ODD were found to have a smaller scleral canal size in comparison with the control group [2]. Moreover, studies comparing patients with 
Fig. 2 Sector representation of the detection of ODD and PHOMS with EDI-OCT (first row). Co-occurrence of ODD/ PHOMS and same sector thinning of RNFL (second row). A grey shade is present in the sectors were a statistically significant $(p \leq 0.05)$ association was found between the presence of ODD and decreased RNFL thickness. Abbreviations:

$\mathrm{T}$ temporal sector, TS superotemporal sector, NS superonasal sector, $\mathrm{T}$ temporal sector, N nasal sector, NI inferonasal sector,

TI inferotemporal sector, EDIOCT enhanced depth imaging optical coherence tomography, ODD optic disc drusen, PHOMS peripapillary hyperreflective ovoid mass-like structures, RNFL, retinal nerve fibre layer

\section{EDI-OCT}
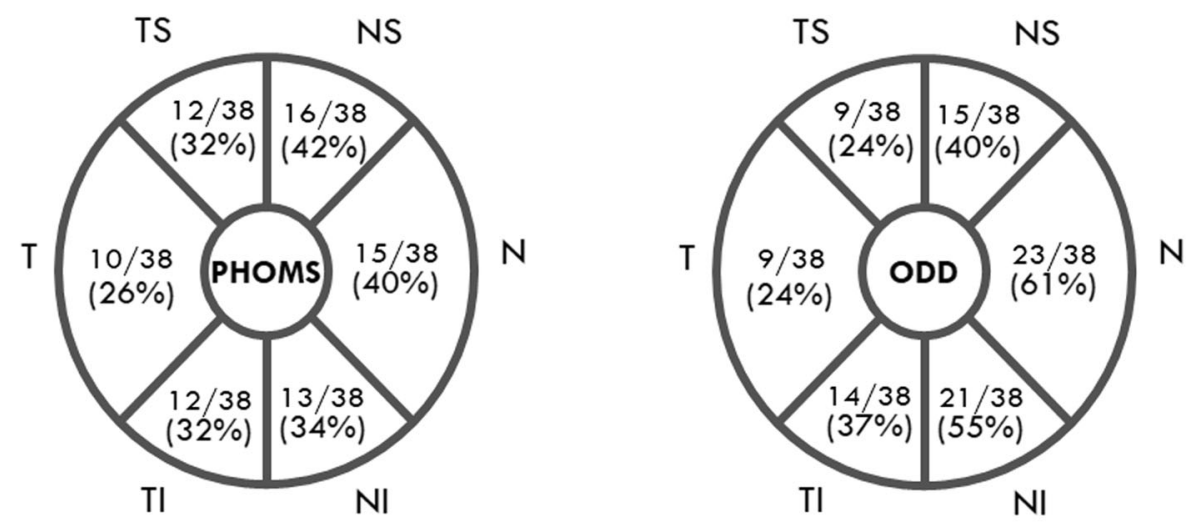

RNFL

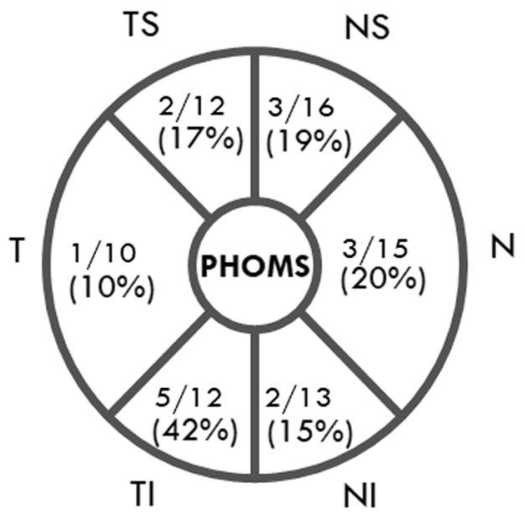

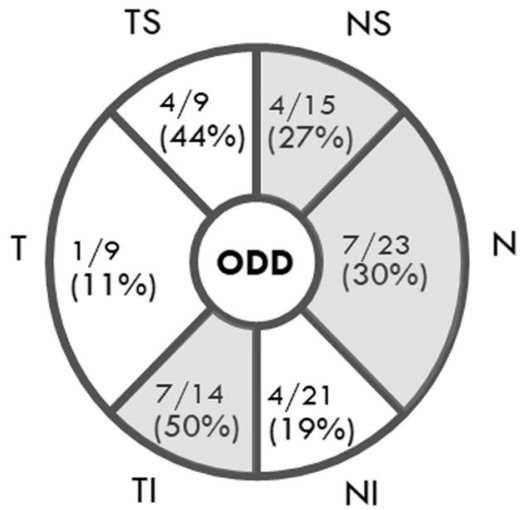

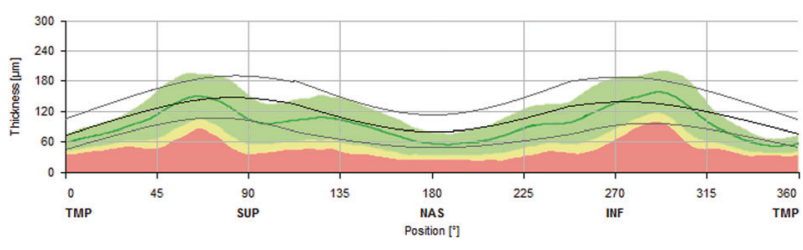

Fig. 3 Retinal nerve fibre layer (RNFL) thickness OCT diagram. Green line: general population RNFL thickness. Black middle line: mean thickness of the study population with optic disc drusen; Grey superior line: upper standard deviation thickness; Grey inferior line: lower standard deviation thickness

superficial ODD to patients with buried ODD verified that the superficial drusen trait is also associated with a wider canal $[8,13]$. A possible explanation for this disparity of results might be due to the fact that children with narrower canals associated with other contributing factors will develop ODD. Throughout the years, the ODD accumulation and calcification might contribute to the distention of the canal. This is in agreement with our findings where younger children with buried drusen were found to have smaller mean scleral canal diameter compared with older children with visible drusen $(p<0.01)$. We also believe that drusen might be more common in females due to a narrower scleral canal size compared with males. However, published data do not describe any difference between genders when comparing the scleral canal diameter [13, 21].

Visual field defects tend to occur in older patients and are associated with large and superficial drusen [7]. In this study, although a high percentage of patients did not cooperate in VF testing, VF defects were more common in children with visible ODD (25\%) compared with children with buried ODD (17\%). A 15\% complication rate associated with ODD was reported, being in all cases peripapillary haemorrhages with scotomas in the visual fields. In comparison with a published case series of five children with ODD and peripapillary haemorrhages, symptoms included floaters and blurred vision and only one patient had VF defect [22].

Various imaging exams have been used to identify ODD. Auto-fluorescence is an excellent diagnostic method in adults; however, it is not reliable for buried drusen detection [7]. In our study, the detection rate of ODD with FAF in both groups (visible and buried) was only $50 \%$. Therefore, we find FAF less useful for identifying ODD in 
Table 3 Association between the presence of ODD and PHOMS with RNFL thickness decrease evaluated by $\mathrm{ONH}$ sector

\begin{tabular}{llllll}
\hline Sector & RNFL loss $^{\mathrm{a}}, n(\%)$ & Normal RNFL, $n(\%)$ & ODD, $n$ & Chi $^{2}(\mathrm{df})$ & $p$ value \\
\hline ODD & & & & \\
Nasal & $7(30 \%)$ & $16(70 \%)$ & 23 & $5.60(1)$ & $0.02^{*}$ \\
Superonasal & $4(27 \%)$ & $11(73 \%)$ & 15 & $3.96(1)$ & $0.05^{*}$ \\
Superotemporal & $4(44 \%)$ & $5(56 \%)$ & 9 & $2.81(1)$ & 0.09 \\
Temporal & $1(11 \%)$ & $8(89 \%)$ & 9 & $0.81(1)$ & 0.37 \\
Inferotemporal & $7(50 \%)$ & $7(50 \%)$ & 14 & $4.78(1)$ & $0.03 *$ \\
Inferonasal & $4(19 \%)$ & $17(81 \%)$ & 21 & $1.43(1)$ & 0.23 \\
& & & & & \\
\hline Sector & RNFL loss ${ }^{\mathrm{a}}, n(\%)$ & Normal RNFL, $n(\%)$ & PHOMS, $n$ & Chi (df) & $p$ value \\
\hline PHOMS & & $12(80 \%)$ & 15 & $0.04(1)$ & 0.84 \\
Nasal & $3(20 \%)$ & $13(81 \%)$ & 16 & $0.76(1)$ & 0.38 \\
Superonasal & $3(19 \%)$ & $10(83 \%)$ & 12 & $0.48(1)$ & 0.49 \\
Superotemporal & $2(17 \%)$ & $9(90 \%)$ & 10 & $0.61(1)$ & 0.44 \\
Temporal & $1(10 \%)$ & $7(58 \%)$ & 12 & $2.13(1)$ & 0.14 \\
Inferotemporal & $5(42 \%)$ & $11(85 \%)$ & 13 & $0.90(1)$ & 0.77 \\
Inferonasal & $2(15 \%)$ & & & & \\
\hline
\end{tabular}

$C h i^{2}$ chi-square test for independence, $d f$ degrees of freedom, EDI-OCT enhanced depth imaging optical coherence tomography, PHOMS peripapillary hyperreflective ovoid mass-lie structures, RNFL retinal nerve fibre layer, $O D D$ optic disc drusen

*Statistically significant difference $(p \leq 0.05)$

${ }^{\text {a }}$ Patients categorised with a RNFL loss presented values that were outside or borderline below normal limits children, who are more likely to harbour non-calcified buried drusen.

Previously, US was regarded as the gold standard imaging method for diagnosing ODD, as it detects deeply situated calcium deposits in the $\mathrm{ONH}$ even in uncooperative children $[3,5,23]$. In younger patients some drusen are not yet fully calcified and are consequently undiagnosed by this imaging technique. In this study, ophthalmic US detected $80 \%$ of patients with buried ODD and 100\% with visible ODD.

The EDI-OCT is an imaging technique that provides microscopic resolution of $\mathrm{ONH}$ and has the highest sensitivity near the inner sclera. Thereby it is an excellent method to visualise small structures, such as drusen, and especially buried ODD [8-10]. Recent data establish EDIOCT the gold standard exam in ODD diagnosis as it is able to visualise their structure, size and shape better than any other imaging modalities. It also has a higher detection rate compared with US [5, 11]. In our study all patients had drusen that were identified by EDI-OCT. The diagnostic criteria of ODD using EDI-OCT have been a debate issue for the last few years. After the ODD Consortium, it was established that drusen should be identified as hyporeflective structures with/without a hyperreflective border, that are present above the lamina cribrosa [17]. It has been previously hypothesised, that the hyporeflective appearance of drusen on OCT is due to the highly dense and uniform composition. In the same way that leaking fluid or cysts appear hyporeflective on OCT, dense and homogenous material may appear hyporeflective as well. The hyperreflective border is thought to be due to the presence of calcium in the more superficial area of drusen [14].

The distribution of ODD over ONH sectors has been assessed in several studies, and was found to be more predominant on the nasal side [5, 9]. Likewise, the RNFL defect is typically nasal, with sparing of the temporal side $[9,12,13,24]$. In our study we also found a predominance of ODD on the nasal side. However, contrary to what we would expect, inferotemporal and superotemporal sectors were also commonly affected. These findings might be explained as patients with ODD in the inferotemporal and superotemporal sectors presented the lowest mean scleral canal diameter, although this difference did not achieve statistical significance.

In this cohort of patients, EDI-OCT identified PHOMS in $90 \%$ of the eyes with ODD. The exact origin of PHOMS is still unknown, although there is histopathological evidence that they might correspond to lateral bulging or herniation of distended retinal nerve fibres into the peripapillary retina $[1,5]$. PHOMS can only be detected by OCT as they do not auto-fluoresce, and they are not detected by US. On OCT, PHOMS appear hyperreflective without a sharp outer margin and lie around the optic disc [5]. This OCT pattern has been described in patients without ODD, as in patients with optic nerve tumours [16] or with papilledema [15]. The recent literature suggests that PHOMS were previously misdiagnosed as 'hyperreflective drusen'; however, these structures should not be diagnosed as ODD [5, 17]. In this study, although ODD and PHOMS were found at the same location (nasal, inferonasal and superotemporal) and with 
the same frequency, PHOMS were not associated with RNFL loss. Conversely, we found a significant association between ODD and decreased RNFL thickness in nasal ( $p=$ $0.02)$, superonasal $(p=0.05)$ and inferotemporal $(p=0.03)$ sectors. Therefore, our results show that only ODD are causing compression over RNFL causing thickness reduction, and PHOMS do not have an effect upon this layer. This finding might be new supporting evidence for considering PHOMS as 'secondary effect' of ODD. We are in agreement with the theory that hyperreflective masses around the ONH should not be considered as ODD and should be named as PHOMS.

This study presents several limitations. We acknowledge the limited number of our sample population can make generalisation of the obtained results difficult. Moreover, non-probability sampling of participants can lead to biased interpretation of the results. In addition, we did not perform EDI-OCT with dense ONH scans in both vertical and horizontal direction. Only the star pattern was performed to reduce scanning time and facilitate children's cooperation. Finally, we would also like to address that currently there is no normative database for RNFL thickness in children, hence adult reference values were used for this analysis.

In conclusion, this study showed that EDI-OCT is a valuable tool for morphological analysis of ODD and PHOMS in children. Buried drusen, which are the most common in children and are difficult to detect with other imaging modalities, were easily diagnosed using EDI-OCT. Moreover, our findings represent a new supporting argument for diagnosing hyperreflective masses around the optic disc as PHOMS, as they are distinct from drusen in both appearance and impact on RNFL. Drusen are hyporeflective and appear on the ONH above lamina cribrosa and were associated with decreased RNFL thickness. On the contrary, PHOMS are hyperreflective structures located around the $\mathrm{ONH}$ and were not associated with RNFL loss.

\section{Summary}

\section{What was known before}

- Optic disc drusen on OCT are considered to be hyporeflective masses on the optic nerve head. Peripapillary hyperreflective ovoid mass structures (PHOMS) are thought to be a bulging of the nerve fibre layer due to the presence of drusen. Although previously they have been classified as hyperreflective drusen.

- EDI-OCT is now considered the gold standard for diagnosing optic disc drusen.

- In children, OCT is used to diagnose optic disc drusen. Although no specific study was conducted analysing children with optic disc drusen and EDI-OCT.

\section{What this study adds}

- EDI-OCT showed to be a valuable tool analysing optic disc drusen in the paediatric population. Allowing the comparison between children with buried and ophthalmoscopic visible drusen.

- The presence of optic disc drusen was associated with a decreased thickness of the RNFL within the same location. On the contrary the presence of PHOMS was not related to a decrease in thickness of the RNFL. This finding might be a new supporting argument for diagnosing hyperreflective masses around the $\mathrm{ONH}$ as PHOMS, and not as hyperreflective drusen as previously described in the literature.

\section{Compliance with ethical standards}

Conflict of interest The authors declare that they have no conflict of interest.

Publisher's note Springer Nature remains neutral with regard to jurisdictional claims in published maps and institutional affiliations.

\section{References}

1. Mo T. Pathology and pathogenesis of drusen of the optic nerve head. Ophthalmology. 1981;88:1066-80.

2. Malmqvist L, Li XQ, Eckmann CL, Skovgaard AM, Olsen EM, Larsen $\mathrm{M}$, et al. Optic disc drusen in children: the copenhagen child cohort 2000 eye study. J Neuro-Ophthalmol. 2017;0:1-7.

3. Auw-Haedrich C, Staubach F, Witschel H. Optic disk drusen. Surv Ophthalmol. 2002;47:515-32.

4. Kiegler HR. Comparison of functional findings with results of standardized echography of the optic nerve in optic disk drusen. Wien Klin Wochenschr. 1999;107:651-3.

5. Hamann S, Malmqvist L, Costello F. Optic disc drusen: understanding an old problem from a new perspective. Acta Ophthalmol. 2018;1:1-12.

6. Pineles SL, Arnold AC. Fluorescein angiographic identification of optic disc drusen with and without optic disc edema. J Neuroophthalmol. 2012;32:17-22.

7. Chan MY, Pineles SL. Optic disc drusen in children. Surv Ophthalmol. 2016;61:745-58.

8. Malmqvist L, Lindberg ASW, Dahl VA, Jørgensen TM, Hamann S. Quantitatively measured anatomic location and volume of optic disc drusen: an enhanced depth imaging optical coherence tomography study. Investig Ophthalmol Vis Sci. 2017;58:2491-7.

9. Sato T, Mrejen S, Spaide RF. Multimodal imaging of optic disc drusen. Am J Ophthalmol. 2013;156:275-.e1.

10. Silverman AL, Tatham AJ, Medeiros FA, Weinreb RN. Assessment of optic nerve head drusen using enhanced depth imaging and swept source optical coherence tomography. J Neuroophthalmol. 2014; 34:198-205.

11. Merchant KY, Su D, Park SC, Qayum S, Banik R, Liebmann $\mathrm{JM}$, et al. Enhanced depth imaging optical coherence tomography of optic nerve head drusen. Ophthalmology. 2013;120: 1409-14. 
12. Lee KM, Woo SJ, Hwang JM. Morphologic characteristics of optic nerve head drusen on spectral-domain optical coherence tomography. Am J Ophthalmol. 2013;155:1139-.e1.

13. Flores-Rodríguez P, Gili P, Martín-Ríos MD. Ophthalmic features of optic disc drusen. Ophthalmologica. 2012;228:59-66.

14. Slotnick S, Sherman J. Buried disc drusen have hypo-reflective appearance on SD-OCT. Optom Vis Sci. 2012;89:704-8.

15. Paton L, Holmes G. The pathology of papilloedema: a historical study of sixty eyes. Brain. 1911;33:289-432.

16. Lee KM, Hwang JM, Woo SJ. Optic disc drusen associated with optic nerve tumors. Optom Vis Sci. 2015;92:S67-S75.

17. Malmqvist L, Bursztyn L, Costello F, Digre K, Fraser JA, Fraser $\mathrm{C}$, et al. The optic disc drusen studies consortium recommendations for diagnosis of optic disc drusen using optical coherence tomography. J Neuro-Ophthalmol. 2018; 38:299-307.

18. Langenegger SJ, Funk J, Toteberg-Harms M. Reproducibility of retinal fiber layer thickness measurements using eye tracker and the retest function of Spectralis SD-OCT in glaucomatous and healthy control eyes. Invest Ophthalmol Vis Sci. 2011;52:3338-44.

19. Seitz R, Kersting G. Die drusen der Sehnerven-papille und des pigmentepithels. Klin Monatsbl Augenheilkd. 1962;140:75-88.

20. Wallman J, Winawer J. Homeostasis of eye growth and the question of myopia. Neuron. 2004;43:447-68.

21. Floyd MS, Katz BJ, Digre K. Measurement of the scleral canal using optical coherence tomography in patients with optic nerve drusen. Am J Ophthalmol. 2005;139:664-9.

22. Lee KM, Hwang JM, Woo SJ. Hemorrhagic complications of optic nerve head drusen on spectral domain optical coherence tomography. Retina. 2014;34:1142-8.

23. Kurz-Levin MM, Landau K. A comparison of imaging techniques for diagnosing drusen of the optic nerve head. Arch Ophthalmol. 1999;117:1045-9.

24. Noval S, Visa J, Contreras I. Visual field defects due to optic disk drusen in children. Graefes Arch Clin Exp Ophthalmol. 2013;251:2445-50. 